\title{
Quels critères pour l'examen de spécialiste? (état 2007)
}

\author{
La Commission pour la formation postgraduée et continue (CFPC) introduit \\ au $1^{\text {er }}$ janvier 2007 le caractère éliminatoire de l'examen de spécialiste aussi \\ pour les formations approfondies en chirurgie vasculaire, chirurgie thoracique \\ et phoniatrie.
}

\section{Christoph Hänggeli}

Administrateur responsable du Secrétariat pour la formation prégraduée, postgraduée et continue (FPPC)
La CFPC a mis en vigueur au $1^{\text {er }}$ janvier 2007 la clause éliminatoire de l'examen de formation approfondie pour trois nouvelles spécialités. Ainsi, la réussite de l'examen de spécialiste resp. de la formation approfondie est désormais exigée pour presque tous les titres et une partie des formations approfondies, sous réserve des dispositions transitoires applicables.

\section{Dispositions transitoires}

Font exception les candidats remplissant une des deux conditions suivantes:

- les candidats ayant déjà participé à un examen de spécialiste ou un examen pour une formation approfondie (entièrement ou en partie) avant l'entrée en vigueur du caractère éliminatoire de l'examen ne devront pas passer d'autre examen;

- les candidats terminant leur formation postgraduée dans les deux ans après l'entrée en vigueur du caractère éliminatoire de l'examen de spécialiste ne doivent attester, pour l'obtention du titre ou de la formation approfondie, que leur participation à l'examen. Attention: toutes les conditions doivent être remplies durant les deux ans qui suivent la date d'entrée en vigueur (y compris l'examen de spécialiste ou l'examen de formation approfondie complet, sauf si la société de discipline médicale refuse la participation à la $2^{\mathrm{e}}$ partie de l'examen en raison d'un résultat insuffisant à la $1^{\text {re }}$ partie)!

Pour les disciplines ci-après, seule la participation à l'examen continue d'être exigée, en d'autres termes: il n'est pas nécessaire d'avoir réussi l'examen pour obtenir le titre de spécialiste.

- Chirurgie cardiaque et vasculaire thoracique;

- Chirurgie maxillo-faciale;

- Génétique médicale;

- Néphrologie.
Correspondance:

Christoph Hänggeli

FMH

Secrétariat pour la formation

prégraduée, postgraduée

et continue (FPPC)

Elfenstrasse 18

CH-3000 Berne 15

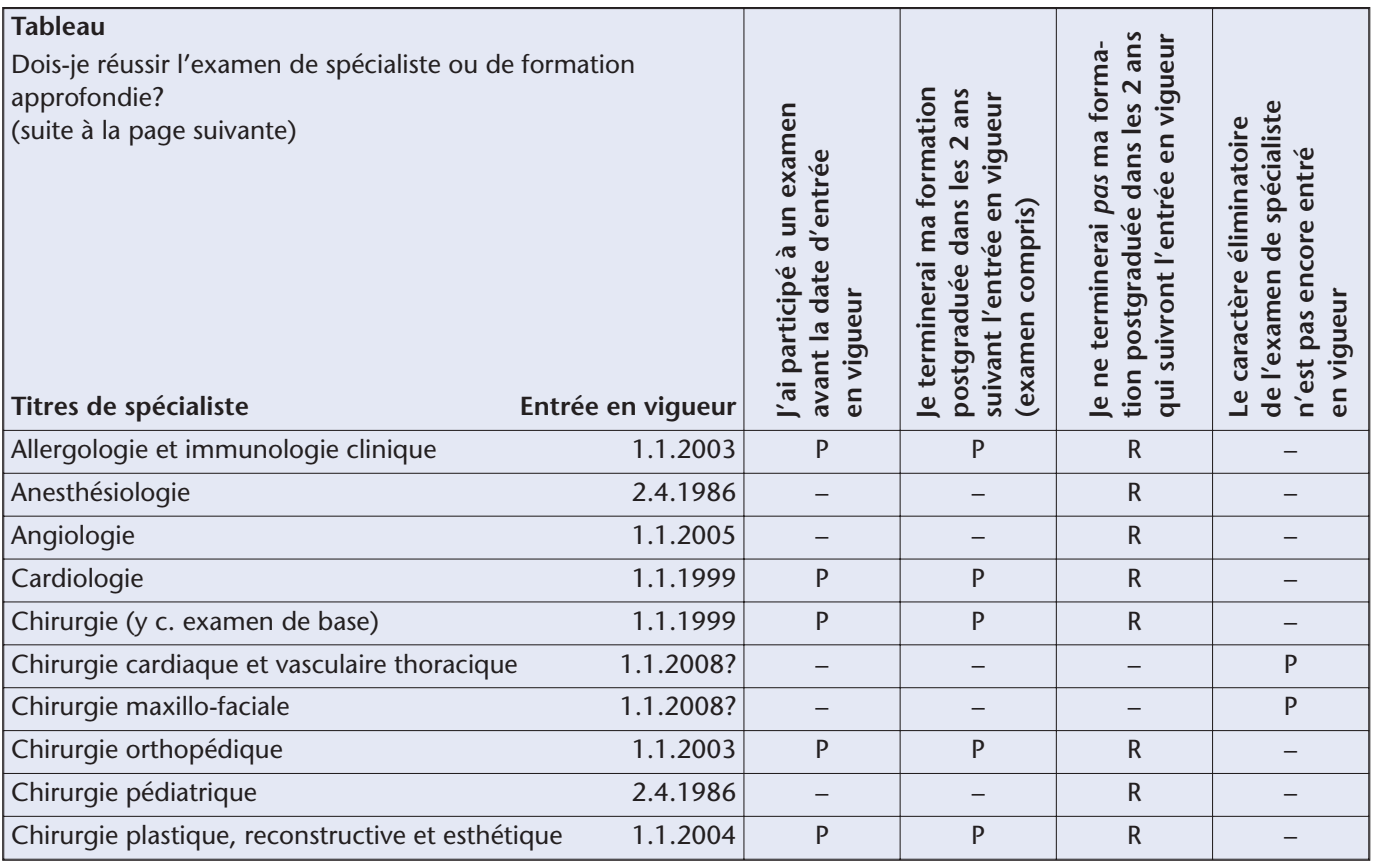


La documentation relative à la

formation postgraduée (avec les dates actuelles d'examen)

se trouve sur le site internet de la FMH à l'adresse suivante: www.fmh.ch/awf. Les dates d'examen sont aussi publiées dans le Bulletin des médecins suisses (BMS). Pour de plus amples informations, veuillez vous adresser au Secrétariat de la formation prégraduée, postgraduée et continue (FPPC) de la FMH, case postale 170 , Elfenstr. 18,3000 Berne 15 , tél. 03135911 11, fax 0313591112 , e-mail: diplome@fmh.ch.

\begin{tabular}{|c|c|c|c|c|c|}
\hline \multicolumn{2}{|c|}{ Tableau } & \multirow[b]{2}{*}{ 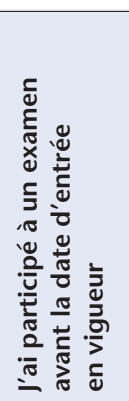 } & \multirow[b]{2}{*}{ 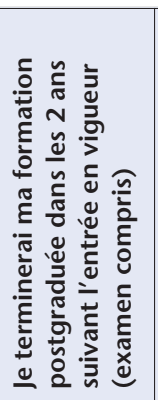 } & \multirow[b]{2}{*}{ 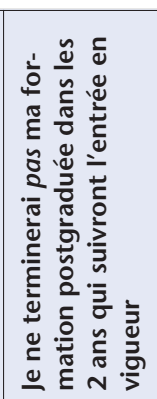 } & \multirow[b]{2}{*}{ 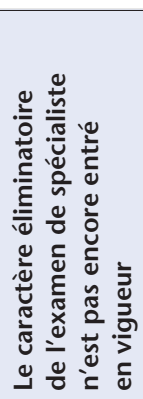 } \\
\hline $\begin{array}{l}\text { Dois-je réussir l'examen de spécialiste ou } \\
\text { approfondie? } \\
\text { (suite de la page précédente) }\end{array}$ & le formation & & & & \\
\hline Dermatologie et vénéréologie & 1.1 .2003 & $\mathrm{P}$ & $\mathrm{P}$ & $\mathrm{R}$ & - \\
\hline Endocrinologie-diabétologie & 1.1 .2001 & $P$ & $P$ & $\mathrm{R}$ & - \\
\hline Gastro-entérologie & 1.1 .2000 & $\mathrm{P}$ & $\mathrm{P}$ & $\mathrm{R}$ & - \\
\hline Génétique médicale & $1.1 .2008 ?$ & - & - & - & $P$ \\
\hline Gynécologie et obstétrique & 1.1 .1999 & $P$ & $\mathrm{P}$ & $\mathrm{R}$ & - \\
\hline Hématologie & 1.1 .2001 & $P$ & $\mathrm{P}$ & $\mathrm{R}$ & - \\
\hline Infectiologie & 1.1 .2003 & $\mathrm{P}$ & $\mathrm{P}$ & $\mathrm{R}$ & - \\
\hline Médecine du travail & 1.1 .2001 & $P$ & $P$ & $\mathrm{R}$ & - \\
\hline Médecine générale & 1.1 .2000 & $P$ & $P$ & $\mathrm{R}$ & - \\
\hline Médecine intensive & 1.1 .2001 & $P$ & $P$ & $\mathrm{R}$ & - \\
\hline Médecine interne & 1.1 .1999 & $\mathrm{P}$ & $\mathrm{P}$ & $\mathrm{R}$ & - \\
\hline Médecine légale & 1.1 .2001 & $P$ & $P$ & $\mathrm{R}$ & - \\
\hline Médecine nucléaire & 2.4 .1986 & - & - & $\mathrm{R}$ & - \\
\hline Médecine pharmaceutique & 1.1 .2003 & $P$ & $P$ & $\mathrm{R}$ & - \\
\hline Médecine physique et réadaptation & 1.1 .2003 & $P$ & $\mathrm{P}$ & $\mathrm{R}$ & - \\
\hline Médecine tropicale & 1.1 .2001 & $\mathrm{P}$ & $P$ & $\mathrm{R}$ & - \\
\hline Néphrologie & $1.1 .2008 ?$ & - & - & - & $\mathrm{P}$ \\
\hline Neurochirurgie & 2.4 .1986 & - & - & $\mathrm{R}$ & - \\
\hline Neurologie & 1.1 .2003 & $\mathrm{P}$ & $\mathrm{P}$ & $\mathrm{R}$ & - \\
\hline Oncologie médicale & 1.1 .2001 & $P$ & $P$ & $\mathrm{R}$ & - \\
\hline Ophtalmologie & 1.1 .2002 & $P$ & $\mathrm{P}$ & $\mathrm{R}$ & - \\
\hline ORL (sans la chirurgie maxillo-faciale) & 1.1 .2001 & $P$ & $P$ & $\mathrm{R}$ & - \\
\hline Pathologie & 1.1 .1999 & $\mathrm{P}$ & $\mathrm{P}$ & $\mathrm{R}$ & - \\
\hline Pédiatrie & 1.1 .2004 & $\mathrm{P}$ & $P$ & $\mathrm{R}$ & - \\
\hline Pharmacologie et toxicologie cliniques & 1.1 .2006 & $\mathrm{P}$ & $\mathrm{P}$ & $\mathrm{R}$ & - \\
\hline Pneumologie & 1.1 .2003 & $\mathrm{P}$ & $\mathrm{P}$ & $\mathrm{R}$ & - \\
\hline Prévention et santé publique & 1.1 .2004 & $P$ & $P$ & $\mathrm{R}$ & - \\
\hline Psychiatrie et psychothérapie & 1.1 .2001 & $P$ & $P$ & $\mathrm{R}$ & - \\
\hline Psychiatrie et psychothérapie d'enfants et $d$ & adolescents 1.1.2002 & $\mathrm{P}$ & $P$ & $\mathrm{R}$ & - \\
\hline Radiologie & 2.4 .1986 & - & - & $\mathrm{R}$ & - \\
\hline Radio-oncologie/radiothérapie & 2.4 .1986 & - & - & $\mathrm{R}$ & - \\
\hline Rhumatologie & 1.1 .2001 & $\mathrm{P}$ & $\mathrm{P}$ & $\mathrm{R}$ & - \\
\hline Urologie & 1.1 .2002 & $\mathrm{P}$ & $\mathrm{P}$ & $\mathrm{R}$ & - \\
\hline \multicolumn{6}{|l|}{ Formations approfondies } \\
\hline Cardiologie pédiatrique & 1.1 .2001 & $\mathrm{P}$ & $P$ & $\mathrm{R}$ & - \\
\hline Chirurgie thoracique & 1.1 .2007 & $P$ & $P$ & $\mathrm{R}$ & - \\
\hline Chirurgie vasculaire & 1.1 .2007 & $\mathrm{P}$ & $\mathrm{P}$ & $\mathrm{R}$ & - \\
\hline Endocrinologie-diabétologie pédiatrique & 1.1 .2005 & $\mathrm{P}$ & $\mathrm{P}$ & $\mathrm{R}$ & - \\
\hline Gériatrie & 1.1 .2006 & $P$ & $P$ & $\mathrm{R}$ & - \\
\hline Néonatologie & 1.1 .2003 & $P$ & $P$ & $\mathrm{R}$ & - \\
\hline Neuropédiatrie & 1.1 .2003 & $\mathrm{P}$ & $\mathrm{P}$ & $\mathrm{R}$ & - \\
\hline Phoniatrie & 1.1 .2007 & $P$ & $P$ & $\mathrm{R}$ & - \\
\hline Radiologie pédiatrique & 1.1 .2006 & $\mathrm{P}$ & $\mathrm{P}$ & $\mathrm{R}$ & - \\
\hline Toutes les autres formations approfondies & & - & - & - & $P$ \\
\hline
\end{tabular}

